suffering from classical chorea. Removal of the tonsils and rest in bed soon restored her to perfect health.

Group 4.-Other things may suffice to relieve hyperthyroidism, rather than resort to the surgery of the thyroid gland.

CASE 8.-Mrs. B., a housewife, aged 23, mother of two children, had had goiter for several years. When I saw her, the symptoms were tachycardia, tremors, an enlarged tinyroid, von Graefe's sign, sweating, emotionalism, insomnia, a phobia of being alone, etc. The tonsils, which had given trouble for years, were removed. After two weeks in bed and a trip to the country for six weeks, she was markedly improved.

CASE 9.-L., a barber, aged 20 , had the ordinary symptoms of hyperthyroidism. He had suffered with tonsillitis every winter since childhood. His tonsils were removed and he was soon able to go on with his work without any further thyroid disturbance.

Group 5.-Let us beware of that old hobbyhorse we have so often ridden-a nervous breakdown from overwork. If we look closely, we shall find something else to account for the patient's condition.

CASE 10.-Dr. D., aged 39, after a rather careful examination, had been sent to the seashore with his wife to recover from "overwork." His nervousness had increased and when I saw him, general paresis was easily diagnosed. He afterward died in the insane asylum.

CASE 11.-B., a traveling man, aged 41, was considered overworked, notwithstanding his robust physical condition. The symptoms were irregular pupils, insomnia, depressed spirits and a ++++ Wassermann reaction. It was decided best to send him to an institutior. for treatment and confinement.

Group 6.-The last and largest group includes cases of oral infection, pyorrhea, and abscessed and carious teeth. Everybody is talking about oral infection and, perhaps, exaggerating its importance.

CASE 12.-Mrs. C., housewife, aged 62 , who had been a rheumatic for years, and was recently found to have high blood pressure, was suddenly seized with anginoid attacks. After about three weeks of waiting for death to claim her and of exhausting a large supply of amyl nitrite pearls, she was sent to a hospital and found to have abscessed teeth and diseased tonsils. These foci were removed, and she became a useful woman. Her intercostal neuralgia is now a harrowing memory to a very good doctor.

CASE 13.-D., aged 45, a robust man of good habits, had severe rheumatic pains and intense, diffuse bronchitis, with asthmatic breathing. He was unable to lie down at night. On examination, he was found to have pyorrhea, with many abscessed teeth. Extraction of the teeth and treatment of the gums for a short time, followed by two weeks at the seashore, completely restored him.

CASE 14.-L., aged 17, a clerk, had been troubled with a number of painful abscesses. An abscessed tooth was extracted. His general health improved and no furunculosis has since occurred.

CASE 15.-O., a man, aged 66, who was in the insurance business, had a well defined arrhythmia. The heart sounds were almost inaudible, and the blood pressure was low. He experienced dyspnea on the slightest exertion, and his liver was enlarged. His spirits were markedly depressed. He was found to have five abscessed teeth, which were removed at two sittings. He has greatly improved, and the normal heart condition has been completely restored.

CASE 16.-C., a man, aged 50, suffered from asthenia, from an intense depression of spirits, that was almost melancholia, from marked insomnia, and loss of appetite. He was practically bedridden. A number of severely abscessed teeth were removed, and he is making a slow but definite recovery.
CASE 17.-C., a man, aged 67, had been troubled with a number of furuncles. He was found to have almost the same symptoms as the patients in the two foregoing cases. All his teeth had to be removed. He insisted on trying to attend to some business, which proceeding made his convalescence slow.

In the Hillman Hospital there are many of the adult and aged "down and out" class, who, in times past, have been healthy and useful citizens. As a rule their mouths and teeth have been grossly neglected. Their teeth are irregular, loose, carious and broken, and set in gums seething with pus. I have often asled myself the question, "Is there any connection between oral infection and being 'down and out'?"

Then comes the practical problem, What are we going to do about it? Prevention, by teaching oral hygiene, will help the future generation. But our own patients must be taken care of. Shall we deal with this pathologic condition radically or conservatively? Many deaths have been reported from too radical treatment. Apparently the best results are obtained by putting the patient to bed, clearing up the pathologic condition a little at each sitting, allowing from three to ten days between treatments, according to the amount of reaction and recuperation of the patient.

Is oral infection a passing fad? Perhaps we are reading too much into it. It is certainly easy to become too enthusiastic. To me it is one of the present problems in medicine, extending into all the specialties. We should, however, remember the plea to view the whole patient as a diagnostic problem. If we work and watch while waiting, oral infection will find its proper place in medicine.

\section{CHRONIC SEPTICEMIC ENDOCARDITIS}

WITH SPLENOMEGALY TREATMENT BY SPLENECTOMY

$$
\begin{aligned}
& \text { DAVID RIESMAN, M.D. } \\
& \text { PHILADELPHIA }
\end{aligned}
$$

In the face of a disease like cancer or sarcoma we accept our helplessness with resignation; but we chafe against our helplessness in so-called malignant endocarditis, for we feel that we should be able to master it, the disease being an infective process due to well known bacteria, which in other circumstances may be successfully combated. Dr. Libman has shown that patients suffering from this type of endocarditis may become bacteria free and yet succumb to the disease. If death were an early event, the failure of our efforts might be more readily understood; but the affection may last for months or even for one or two years, so that ample time exists for treating it, and yet nearly every case terminates fatally.

I shall not go into detail regarding the symptoms of the affection, with which the writings of Osler, Dock and Libman have made us familiar. The outstanding features are fever of long duration, which may resemble that of tuberculosis or malaria; anemia of varying intensity, sometimes so severe as to suggest one of the primary anemias; pains in the joints; petechial spots on the skin and mucous membranes; and a heart murmur. Albuminuria is common, and in later stages the signs of a true nephritis may appear. Abdominal pains, sometimes sudden in onset and suggesting embolic processes in the spleen or kidneys, are not infrequent. Cough and insomnia are often very troublesome. From the diagnostic point of view, the 
heart murmur is the most important thing; it may be very faint or it may be loud and distinct. I have seen cases with mitral, with aortic and with pulmonic lesions; on the whole, however, I have gotten the impression that the aortic cases are the most frequent. The affection nearly always attacks a valve previously diseased as the result either of rheumatism or of some allied condition, such as tonsillitis or chorea. It may, however, have its starting point on an intact valve, as I have observed in gonorrhea and in one case of pnet1monia, in which the pulmonic valve became involved. Such cases generally run a more acute course, with higher fever, and do not present so prominently the renal features. There is a primary chronic form of endocarditis, however, which in its course and termination is indistinguishable from that in which the lesion is a secondary one on a previously diseased valve.

One other feature is important, and that is the condition of the spleen. This organ is practically always enlarged, and in some cases so much enlarged that it becomes the dominant feature to the point of completely obscuring the underlying heart affection. It is of this group of cases, those characterized by a true splenomegaly, that I wish especially to speak. The spleen may extend down to the level of the umbilicus or even lower; it is smooth, rather firm and rarely tender, though often the seat of spontaneous pain. Such a striking enlargement may readily lead to a wrong diagnosis, especially to that of splenic anemia or of Banti's disease. It is easy to see why such a diagnosis might be made. There is the prominent spleen, the profound anemia, at times a striking leukopenia; and if a murmur is found, it will often be attributed to the anemia. Petechial hemorrhages, so valuable in the diagnosis, may be entirely absent throughout the long course of the disease.

I am inclined to think that in more than one case of so-called splenic anemia or Banti's disease treated by splenectomy, the condition was in reality chronic septicemic endocarditis. The leukopenia in a disease in which one would expect leukocytosis might readily, though erroneously, tilt the diagnostic scale in favor of splenic anemia.

Watching a number of cases in the last two or three years, and finding that neither autogenous nor stock vaccines, transfusions, drugs or other measures did aught to avert death, I began to wonder whether there was not some other factor besides the heart that was of importance in the fatality of the disease. Baehr, ${ }^{1}$ Löhlein, ${ }^{2}$ Ophüls ${ }^{3}$ and others have called attention to embolic processes in the kidney. While these undoubtedly play an important rôle in the course of the disease, I believe it is wise to direct attention to the possible significance of the large spleen. The spleen is a filter for bacteria. Whether it kills them or only benumbs them, leaving their final execution to the liver, is not definitely known. Bacterial filtration is not a passive process, but according to Rosenow, and to Ozaki, ${ }^{4}$ it is dependent on vital activity of the cells, on a selective action on their part, a bacteriotropism. The filtration causes an accumulation of bacteria in the spleen which leads to hypertrophy, a sort of work hypertrophy, as William Mayo calls it. Through the

1. Baehr: Am. Jour. Med. Sc., September, 1912, p. 327.

2. Iohlein: Arb. a. d path. Inst. zu Leipzig, 1907, No. 4

3. Ophüls, William: Nephritis, THE JourNat A M A. Nov, 13, 1915, p. 1719; The Etiology and Development of Nephritis, Oct. 13, 1917. p. 1223 .

4. Ozaki, Y: Jour. Med. Research, 1917, 36, 413. multiplication of bacteria, and perhaps through their subsequent autolysis, increased quantities of toxins are thrown into the circulation. Moreover, living bacteria are often present in old infarcts of the spleen, as shown by Ophüls. ${ }^{5}$ In these ways an infectious disease may be kept up and perpetuated, although the primary focus is no longer existent or active. An example or two will serve to illustrate this point. In syphilis the spleen at times is greatly enlarged, and in such cases antisyphilitic treatment does little or no good, probably because the spirochetes are well protected in the spleen pulp and tissues. Removal of the spleen brings about a cure, especially if it is followed by a course of antisyphilitic treatment. In chronic malaria the blood is often free from plasmodia, while the spleen shelters them in enormous numbers.

May not therefore the long continued existence of bacteria in the spleen in endocarditis be the principal reason why the so-called bacteria-free cases go on to a fatal termination? If we could get rid of the large disease-harboring focus represented by the spleen, it might be possible to deal with the bacteria present in the heart valves. Enough antibodies might be developed to destroy them; while as long as the spleen continues to pour toxins into the blood, the body, as we know only too well, can never make any headway.

There is another factor to be considered. Enlargement of the spleen is brought about chiefly by a proliferation of cells, to a lesser degree by an increased blood content. This proliferation, according to the theory of cellular toxemia which $I^{6}$ proposed several years ago, causes the pouring into the circulation of enormous quantities of metabolic products that have to be handled by the liver and other organs and tissues.

In view, therefore, of the hopelessness of our treatment of chronic septicemic endocarditis, and in view also of the value of splenectomy in other infectious diseases-as in syphilis, malaria, and in splenic anemia, if that be infectious - I believe we are warranted in removing the spleen in cases of septicemic endocarditis in which the organ is enlarged. In this connection it should be remembered that, as William Mayo ${ }^{7}$ has pointed out, enlargement of the spleen may exist even though the organ cannot be palpated.

On the basis of these thoughts we undertook the operation in one case, and while the ultimate outcome was not all we had hoped, failure was due to an unusual complication which caused the patient's death some time after the operation.

\section{REPORT OF CASE}

I. F., a man, aged 57 , a patient of Dr. H. B. Shmookler, had been failing in health for a year. In the past four months he had a more or less continued fever, associated with joint pains but without joint swellings. His spleen had been enlarging, at first gradually, of late more rapidly. A profound anemia had coincidentally developed. From time to time there had been attacks of sharp pain in the left hypochondrium. At no time had he had any hemorrhage from the nose, mouth or bowel. Twelve years before, following an injury to the head, he began to have epileptiform seizures of jacksonian character, which later gave place to attacks of petit mal. When I sav the patient, Oct. 31, 1917, I found him exceedingly pale and weak. The heart was somewhat enlarged and there was a systolic murmur at the mitral area transmitted into the axilla. This murmur I

5. Ophü1s, William: Nephritis, The Journal A. M. A., Nov. 13, 1915, p. 1719

6. Ricsman, David: The Cellular Factor in Infectious Diseases, Tine Journal A. M. A., Feb. 20, 1915 , p. 649.

7. Mayo, W. J.: Reports from the Mayo Clinic, 1916, 8, 628. 
learned had existed for many years. Petechial spots were present over the legs. The most striking feature, however, was an enlarged spleen which extended to the level of the umbilicus. A blood examination revealed: hemoglobin, 45 per cent.; red cells, 2,220,000; white cells, 4,800, with 82 per cent. polymorphonuclear cells. Considering the case to be one of septicemic endocarditis with splenomegaly, I advised removal of the spleen preceded by transfusion. Two transfusions were done, the second, which was of appreciable benefit, Feb. 3, 1918. February 5, the patient was admitted to the Lankenau Hospital, where an immediate blood conint revealed: hemoglobin, 40 per ccit.; red blood cells, 2,020,000; white blood cells, 4,450; polymorphonuclears, 80 ; lymphocytes, 6; large mononticlears, 8; transitionals, 6; eosinophiles, 0 .

The urine was yellow; of acid reaction; specific gravity, 1.015 , and showed albumin, a faint trace; sugar, none; urea, 1 per cent.; bile, 0 ; indican, 0 ; diacetic acid, 0 ; acetone, 0 ; many granular casts; epithelial cells; white blood cells.

The phenolsulphonephthalein test resulted as follows: the first hour, 7 per cent.; second hour, 12 per cent.; third hour, 14 per cent.; total, 33 per cent.

The blood urea was $66 \mathrm{mg}$. per hundred c.c. of blood.

February 6 , the spleen was removed by Dr. Deaver under ether anesthesia. It was found to be enormously enlarged and adherent to the diaphragm at the upper pole.

The spleen with blood weighed 1,570 gm.; without blood, $1,160 \mathrm{gm}$. The surface showed two scars, evidently the result of infarction. Dr. Reimann, pathologist of the Lankenau Hospital, reported on the microscopic examination: "The spleen is not the spleen of Gaucher's disease, Banti's disease, malaria, tuberculosis, syphilis, Hodgkin's disease, or the leukemias. There is endothelial hyperplasia, diffuse around the blood sinuses, just as in the ordinary acute splenic tumor, e. g., typhoid. The inference is that the patient had a long standing infection of very low virulence or a long standing toxemia."

The day following the operation the hemoglobin rose to 50 per cent. and the red cells to nearly $4,000,000$ from $2,020,000$. At the end of a month the hemoglobin was 55 per cent. and the red cells just under $3,000,000$, and the leukocytes had risen to 6,250 , with 68 per cent. polymorphonuclears instead of 80 per cent.

On the ninth day the stitches were removed, but there was no healing and the patient eviscerated himself. The bowels were reduced and the wound closed under chloroform anesthesia. No infection ensued.

After the operation the patient improved amazingly in appearance; his hands were no longer cold, and he lost the yellowish cachectic pallor which had been such a striking feature. Both Dr. Deaver and Dr. Shmookler feel that the operation justified itself by the improvement that followed.

March 9 , the patient began to have trouble with his larynx and extreme difficulty in breathing. Dr. Butler, on examination, thought there was an abscess of the larynx. Before anything could be done, the man died.

\section{COMMENT.}

The man thus lived a month after the operation; and notwithstanding the fact that he had eviscerated himself and had to have a second operation, his general condition as well as the blood showed a decided improvement. While we do not know what would have happened had the laryngeal abscess not supervened, it is reasonable to suppose that the improvement would have continued.

I have reported this single case in the hope that the operation may be undertaken by others.

1715 Spruce Street.

Overweight at 50.-There is every reason why a man who is overweight at 50 should reduce until he reaches the weight he was when he was 35 . According to Fisk he is a better instrance risk if after 35 he is under the weight which is the average of those of his years.-Lusk, Food in War Time.

\section{A CLINICAL STUDY OF FIVE HUNDRED CASES OF CHOLECYSTITIS}

\section{WITH SPECIAL REFERENCE TO DIAGNOSIS}

\section{W. H. BODENSTAB, M.D. BISMARCK, N. D.}

We live in an age of conservation. Every newspaper we pick up contains articles on the conservation of food, of clothing, of fuel; in fact, we are urged and taught to conserve all the resources of this bountifully productive land, but we see and hear very little on the conservation of the nation's greatest asset, the conservation of human life.

We live in an age of preventive medicine, and although we are not yet able to prevent a great many diseases, we, as physicians and surgeons, frequently are in a position to prevent complications of diseases and through opportune intervention prevent a serious, if not fatal, outcome, thereby doing our bit in the conservation of human life.

While looking over the mortality records for the last five years, and the causes of death in our clinic at Bismarck, I was impressed with the fact that a large percentage of these deaths were preventable, provided these patients had received earlier attention. Some died without operation, while others submitted to surgery as the only possible means of recovery. I refer particularly to the acute intra-abdominal lesions, namely, appendicitis, gastric and duodenal ulcers, and cholecystitis.

We are all familiar with the importance of early diagnosis and surgical treatment of acute appendicitis and perforating gastric ulcer, but I do not think that the profession at large has been strongly enough impressed with the importance of early operations for the relief of gallbladder inflammations and their results. It is especially cholelithiasis and active cholecystitis to which I wish to call attention, not only because of the suffering they entail, but also because of the more serious consequences which have not been sufficiently emphasized and which swell our mortality rate, namely, cancer of the gallbladder or ducts, rupture of the gallbladder with septic peritonitis, empyema of the gallbladder, suppurative cholangeitis, abscess of the liver, hepatitis, pancreatitis and other less serious complications, all of which can be averted by an early diagnosis and operative treatment.

The practice of waiting for further developments, for the appearance of jaundice to verify the diagnosis, or until direct danger to life is evident, is still too general.

Realizing that many lives are thus sacrificed that might be saved, and that the voice of the internist is sometimes heeded by the general practitioner and the patient more than that of the surgeon, I thought that this report might be of help to those who are still holding back, as well as to those on whose diagnostic skill and judgment depend the welfare and the lives of a great many people. I have, therefore, reviewed the histories of the last 500 cases of inflammation of the gallbladder in which operation was performed in our clinic either by Dr. Quain or by Dr. Ramstad, and have tabulated the symptoms as they were recorded at the time these histories were taken and the patients referred for operation. According to Table 1 it will be seen that the diagnosis of gallbladder disease rests almost entirely with the anamnesis, and the patient will 